\title{
A Nonintrusive IGBT Open-Circuit Fault and Current Sensor Fault Diagnosis Method for Grid- Tied Three-phase Three-Wire Inverter with Two Current Sensors
}

\author{
Zhan Li \\ College of Electrical Engineering \\ Zhejiang University \\ Hangzhou, China \\ School of Electrical \& Electronic \\ Engineering \\ Nanyang Technological University \\ Singapore \\ lizhan@zju.edu.cn \\ Zhihong Bai \\ College of Electrical Engineering \\ Zhejiang University \\ Hangzhou, China \\ baizhihong@zju.edu.cn
}

\author{
Pat Wheeler \\ Department of Electrical \\ \& Electronic Engineering \\ University of Nottingham \\ Nottingham, UK \\ pat.wheeler@nottingham. \\ ac.uk
}

Xin Zhang

School of Electrical \&

Electronic Engineering

Nanyang Technological

University

Singapore

jackzhang@ntu.edu.sg

\author{
Alan Watson \\ Department of Electrical \& \\ Electronic Engineering \\ University of Nottingham \\ Nottingham, UK \\ alan.watson@nottingham.ac.uk
}

School of Electrical \&

Electronic Engineering

Nanyang Technological

University

Singapore

e180061@e.ntu.edu.sg

\author{
Alessandro Costabeber \\ Department of Electrical \& \\ Electronic Engineering \\ University of Nottingham \\ Nottingham, UK \\ alessandro.costabeber@nottingh \\ am.ac.uk
}

$\mathrm{Hao} \mathrm{Ma*}$

College of Electrical

Engineering

Zhejiang University

Hangzhou, China

mahao@zju.edu.cn

\begin{abstract}
In some cases, only two current sensors are available in three-phase three-wire (3P3W) inverters. This occurs when one of three sensors is faulty, or the inverter is equipped with only two sensors to save cost. There has been no method addressing the diagnosis of both IGBT open-circuit faults and current sensor faults in the $3 \mathrm{P} 3 \mathrm{~W}$ inverter with only two current sensors. In order to solve this problem, a nonintrusive method based on average output voltage deviations is proposed. The deviations between the measured and estimated output line and phase voltages are utilized to detect and locate the fault. With average model, this method can diagnose the fault fast with only signals available in the controller. Therefore, no extra sampling or diagnosing circuits are needed. Besides, the error-adaptive thresholds are adopted to ensure the robustness. Finally, experimental results verified the effectiveness.
\end{abstract}

Keywords-current sensor fault, diagnosis, IGBT fault, inverter

\section{INTRODUCTION}

Inverters are widely used in renewable energy systems, electrical tractions systems and so on. In inverters, IGBTs are one of the most vulnerable devices [1]. IGBT faults mainly include short-circuit (SC) faults and open-circuit (OC) faults. Both faults may cause the system malfunction. Compared with SC faults, OC faults are less catastrophic and meanwhile are more difficult to be detected. A lot of papers have been published focusing on IGBT OC fault diagnosis. These

This work was supported by the National Nature Science Foundation of China under Grant 51337009 . methods are mainly voltage signal based [2]-[4], current signal based [5]-[7] and model based [8]-[10].

Inverters may also suffer from sensor faults [11]. Sensor faults may cause currents or voltages to rise fast due to closeloop control, which may cause further damages on other devices and loads. Therefore, it is necessary to diagnose sensor fault timely as well. There are some reports on diagnosis of sensor faults. Most of them are based on observers [12]-[14] and current analysis [15]-[16].

However, all these methods mentioned above only consider IGBT faults or sensor fault. The method considering

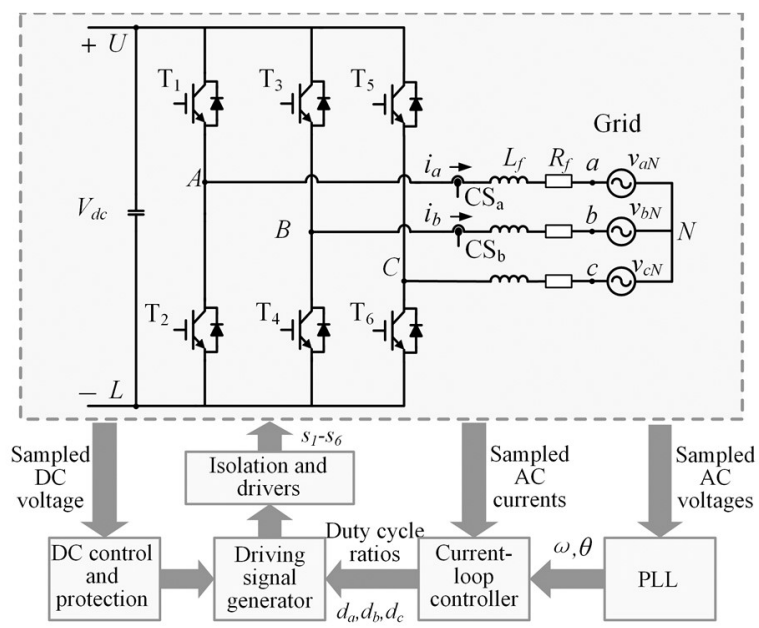

Fig. 1. The grid-tied 3P3W inverter with two current sensors 
only one kind of fault may diagnose falsely when the other kind of fault occurs.

In recent years, some methods have been developed to address both IGBT fault and sensor fault [17]-[18]. In [17], the current deviations generated by a Luenberger observer are used to diagnose both IGBT OC faults and current sensor faults. In order to improve diagnosis speed, [18] proposes a method based on average bridge arm pole-to-pole voltage deviations. In both methods, the sum of three phase currents are used to distinguish IGBT faults from current sensor faults. Hence, these methods are only suitable for three-phase threewire $(3 \mathrm{P} 3 \mathrm{~W})$ inverter with three current sensors.

The literature review shows the problem of diagnosing both IGBT OC faults and current sensors fault in the 3P3W inverters with only two current sensors has not been investigated. For the circumstance where only two current sensors are available in the $3 \mathrm{P} 3 \mathrm{~W}$ inverters, a novel method based on the average output voltage deviations are proposed to diagnose both kinds of faults. The method is nonintrusive, which means the method needs only existing signals in the controller, therefore no extra sampling or diagnosis circuits are added. The method can be embedded in the system easily.

\section{FAULTy Characteristics OF OUtPUt Voltage DEVIATIONS}

Fig. 1 shows the grid-tied 3P3W inverter with only two current sensors. In this paper, a inverter with current sensors in phase $\mathrm{A}$ and phase $\mathrm{B}$ is taken as an example. In the inverter, two phase currents, three phase grid voltages and DC voltage are sampled every switching periods for control. According to the loop shown in Fig.2 and Kirchoff law, the output line voltages $v_{x y}(x, y=a, b, c)$ is

$$
v_{x y}=-L_{f} \frac{d i_{x}}{d t}-R_{f} i_{x}+V_{X Y}+L_{f} \frac{d i_{y}}{d t}+R_{f} i_{y}
$$

Where $X, Y=A, B, C$. Based on (1), the output line voltages can be estimated as

$$
v_{x y}{ }^{*}=-L_{f} \frac{d i_{x}^{\wedge}}{d t}-R_{f} i_{x}^{\wedge}+V_{X Y}{ }^{*}+L_{f} \frac{d i_{y}^{\wedge}}{d t}+R_{f} i_{y}^{\wedge}
$$

Where $i_{x}^{\wedge}$ is the sampled phase current, and $V_{X Y}{ }^{*}$ is the estimated bridge arm pole-to-pole voltage.

Define deviation as

$$
\Delta y=y^{*}-y \text { and } \Delta y=y^{\wedge}-y
$$

Where, $y$ is the real value, $y^{*}$ and $y^{\wedge}$ are the estimated and sampled values respectively. Then, (2) minus (1) gives the output line voltage deviation as

$$
\Delta v_{x y}=-L_{f} \frac{d \Delta i_{x}}{d t}-R_{f} \Delta i_{x}+\Delta V_{X Y}+L_{f} \frac{d \Delta i_{y}}{d t}+R_{f} \Delta i_{y}
$$

Similarly, according to the loop shown in Fig.3, the output phase voltage deviation can be obtained as

$$
\Delta v_{x N}=\Delta V_{X L}-L_{f} \frac{d \Delta i_{x}}{d t}-R_{f} \Delta i_{x}-\Delta V_{N L}
$$

Where

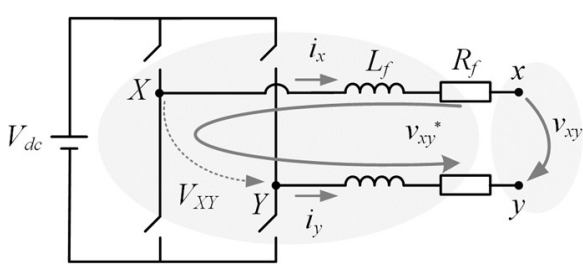

Fig. 2. Loop for calculating output line voltages

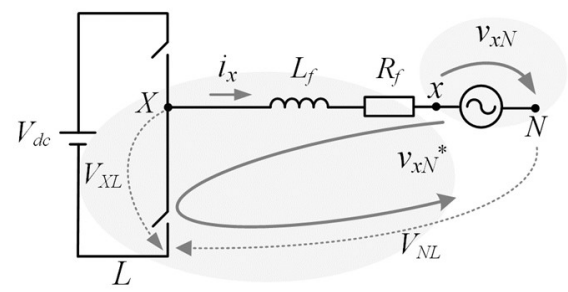

Fig. 3. Loop for calculating output phase voltages

TABLE I. OUTPUT VOLTAGE DEVIATION CHARACTERISTICS OF DIFFERENT IGBT AND CURRENT SENSOR FAULTS IN 3P3W INVERTER WITH TWO CURRENT SENSORS

\begin{tabular}{|c|c|c|c|c|c|c|}
\hline \multirow{2}{*}{ Fault } & \multicolumn{3}{|c|}{$\begin{array}{c}\text { Line voltage } \\
\text { deviations }\end{array}$} & \multicolumn{3}{c|}{$\begin{array}{c}\text { Phase voltage } \\
\text { deviations }\end{array}$} \\
\cline { 2 - 7 } & $\Delta \boldsymbol{v}_{\boldsymbol{a} \boldsymbol{b}}$ & $\Delta \boldsymbol{v}_{\boldsymbol{b} \boldsymbol{c}}$ & $\Delta \boldsymbol{v}_{\boldsymbol{c} \boldsymbol{a}}$ & $\Delta \boldsymbol{v}_{\boldsymbol{a} \boldsymbol{N}}$ & $\Delta \boldsymbol{v}_{\boldsymbol{b} \boldsymbol{N}}$ & $\Delta \boldsymbol{v}_{\boldsymbol{c} N}$ \\
\hline None & $=0$ & $=0$ & $=0$ & $=0$ & $=0$ & $=0$ \\
\hline $\mathrm{T}_{1}$ & $\geq 0$ & $=0$ & $\leq 0$ & $\geq 0$ & $\leq 0$ & $\leq 0$ \\
\hline $\mathrm{T}_{2}$ & $\leq 0$ & $=0$ & $\geq 0$ & $\leq 0$ & $\geq 0$ & $\geq 0$ \\
\hline $\mathrm{T}_{3}$ & $\leq 0$ & $\geq 0$ & $=0$ & $\leq 0$ & $\geq 0$ & $\leq 0$ \\
\hline $\mathrm{T}_{4}$ & $\geq 0$ & $\leq 0$ & $=0$ & $\geq 0$ & $\leq 0$ & $\geq 0$ \\
\hline $\mathrm{T}_{5}$ & $=0$ & $\leq 0$ & $\geq 0$ & $\leq 0$ & $\leq 0$ & $\geq 0$ \\
\hline $\mathrm{T}_{6}$ & $=0$ & $\geq 0$ & $\leq 0$ & $\geq 0$ & $\geq 0$ & $\leq 0$ \\
\hline$\left(\mathrm{CS}_{\mathrm{a}} \times, \mathrm{CS}_{\mathrm{b}}\right)$ & $\neq 0$ & $\neq 0$ & $\neq 0$ & $\neq 0$ & $=0$ & $\neq 0$ \\
\hline$\left(\mathrm{CS}_{\mathrm{a}}, \mathrm{CS}_{\mathrm{b}} \times\right)$ & $\neq 0$ & $\neq 0$ & $\neq 0$ & $=0$ & $\neq 0$ & $\neq 0$ \\
\hline$\left(\mathrm{CS}_{\mathrm{a}} \times, \mathrm{CS}_{\mathrm{c}}\right)$ & $\neq 0$ & $\neq 0$ & $\neq 0$ & $\neq 0$ & $\neq 0$ & $=0$ \\
\hline$\left(\mathrm{CS}_{\mathrm{a}}, \mathrm{CS}_{\mathrm{c}} \times\right)$ & $\neq 0$ & $\neq 0$ & $\neq 0$ & $=0$ & $\neq 0$ & $\neq 0$ \\
\hline$\left(\mathrm{CS}_{\mathrm{b}} \times, \mathrm{CS}_{\mathrm{c}}\right)$ & $\neq 0$ & $\neq 0$ & $\neq 0$ & $\neq 0$ & $\neq 0$ & $=0$ \\
\hline$\left(\mathrm{CS}_{\mathrm{b}}, \mathrm{CS}_{\mathrm{c}} \times\right)$ & $\neq 0$ & $\neq 0$ & $\neq 0$ & $\neq 0$ & $=0$ & $\neq 0$ \\
\hline
\end{tabular}

${ }^{a}\left(\mathrm{CS}_{x} \times, \mathrm{CS}_{y}\right)$ means current sensors $\mathrm{CS}_{x}$ and $\mathrm{CS}_{y}$ are available, where $\mathrm{CS}_{x}$ is faulty.

$$
\Delta V_{N L}=\frac{1}{3}\left(\Delta V_{A L}+\Delta V_{B L}+\Delta V_{C L}\right)
$$

When no fault occurs, $\Delta v_{x y}=0$ and $\Delta v_{x N}=0$. Whereas, when IGBT OC fault or current sensor fault occurs, $\Delta V_{X L} \neq 0$ or $\Delta i_{x} \neq 0$, so $\Delta v_{x y} \neq 0$ and $\Delta v_{x N} \neq 0$. T 1 fault and sensor $\mathrm{CS}_{\mathrm{a}}$ fault are taken as examples to analyze the faulty characteristics of output line voltage deviations and phase voltage deviations.

When $\mathrm{T}_{1}$ is open-circuit, according to analysis in [8], there is $\Delta V_{A L} \geq 0$. Therefore

$$
\Delta v_{a b} \geq 0, \Delta v_{b c}=0, \Delta v_{c a} \leq 0, \Delta v_{a N} \geq 0, \Delta v_{b N} \leq 0, \Delta v_{c N} \leq 0 \text { (7) }
$$

When sensor $\mathrm{CS}_{\mathrm{a}}$ is faulty, there is $\Delta i_{a} \neq 0$. Consequently $\Delta v_{a b} \neq 0, \Delta v_{b c} \neq 0, \Delta v_{c a} \neq 0, \Delta v_{a N} \neq 0, \Delta v_{b N}=0, \Delta v_{c N} \neq 0$ (8) 


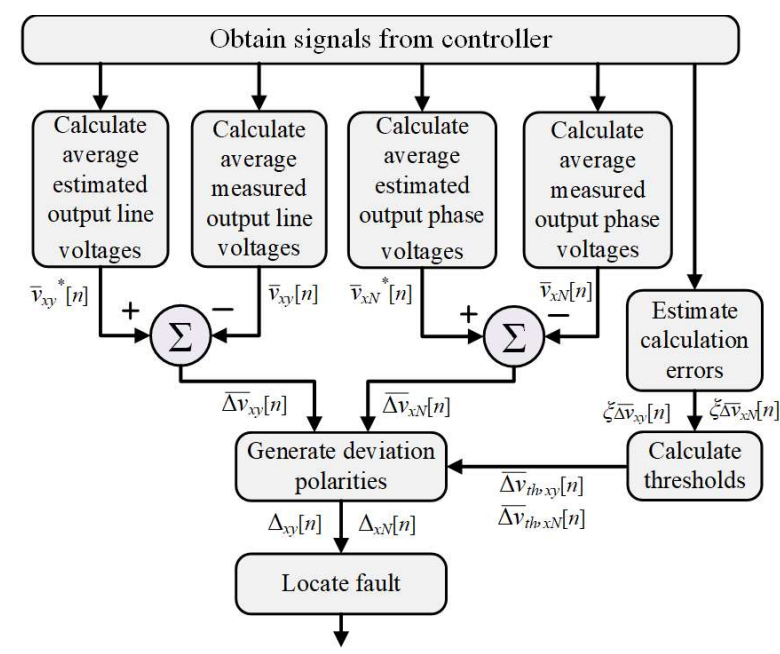

Fig. 4. Principle of the proposed fault diagnosis method

With similar analysis of other IGBT faults and current sensor faults, all faulty characteristics of output voltage deviations for different faults can be concluded in TABLE I.

\section{Proposed Fault Diagnosis Method}

The diagnosis principle is shown in Fig.4. The average output line and phase voltage deviations are used for diagnosis

\section{A. Calculate Average Output Voltage Deviations}

Define average model as

$$
\bar{y}[n]=\frac{1}{T_{S}} \int_{t[n-1]}^{t[n]} y d t
$$

Where $T_{S}$ is the sampling period.

With average model, there is

$$
\begin{aligned}
& \bar{v}_{x y}{ }^{*}[n]=-\frac{L_{f}}{T_{S}}\left(i_{x}^{\wedge}[n]-i_{x}^{\wedge}[n-1]\right)-\frac{R_{f}}{2}\left(i_{x}{ }^{\wedge}[n]+i_{x}^{\wedge}[n-1]\right)+\bar{V}_{X Y}{ }^{*}[n] \\
& +\frac{L_{f}}{T_{S}}\left(i_{y}{ }^{\wedge}[n]-i_{y}{ }^{\wedge}[n-1]\right)+\frac{R_{f}}{2}\left(i_{y}{ }^{\wedge}[n]+i_{y}^{\wedge}[n-1]\right)
\end{aligned}
$$

Where

$$
\bar{V}_{X Y}^{*}[n]=\frac{1}{2}\left(V_{d c}[n-1]+V_{d c}[n]\right)\left(d_{x}[n-1]-d_{y}[n-1]\right)
$$

The voltage sensors are regarded healthy, so the real output line voltage can be obtained as

$$
\bar{v}_{x y}[n]=\frac{1}{2}\left(v_{x y}{ }^{\wedge}[n-1]+v_{x y}{ }^{\wedge}[n]\right)
$$

Similarly, the average estimated and real output line voltages are

$$
\begin{gathered}
\bar{v}_{x N}^{*}[n]=-\frac{L_{f}}{T_{S}}\left(i_{x}^{\wedge}[n]-i_{x}^{\wedge}[n-1]\right) \\
-\frac{R_{f}}{2}\left(i_{x}^{\wedge}[n]+i_{x}^{\wedge}[n-1]\right)+\bar{V}_{X L}^{*}[n]-\bar{V}_{N L}^{*}[n] \\
\bar{v}_{x N}[n]=\frac{1}{2}\left(v_{x N} \hat{\wedge}[n-1]+v_{x N} \hat{}[n]\right)
\end{gathered}
$$

Where
TABLE III. CRITERIA FOR IGBT AND CURRENT SENSOR FAUlT DIAGNOSIS FOR 3P3W INVERTER WITH TWO CURRENT SENSORS

\begin{tabular}{|c|c|c|c|c|c|c|}
\hline \multirow{2}{*}{ Fault } & \multicolumn{3}{|c|}{$\begin{array}{c}\text { Line voltage } \\
\text { deviation polarities }\end{array}$} & \multicolumn{3}{c|}{$\begin{array}{c}\text { Phase voltage } \\
\text { deviation polarities }\end{array}$} \\
\cline { 2 - 7 } & $\boldsymbol{\Delta}_{a b}$ & $\boldsymbol{\Delta}_{\boldsymbol{b c}}$ & $\boldsymbol{\Delta}_{c a}$ & $\boldsymbol{\Delta}_{\boldsymbol{a} \boldsymbol{N}}$ & $\boldsymbol{\Delta}_{\boldsymbol{b} \boldsymbol{N}}$ & $\boldsymbol{\Delta}_{c N}$ \\
\hline None & $\mathrm{Z}$ & $\mathrm{Z}$ & $\mathrm{Z}$ & $\mathrm{Z}$ & $\mathrm{Z}$ & $\mathrm{Z}$ \\
\hline $\mathrm{T}_{1}$ & $\mathrm{P}$ & $\mathrm{Z}$ & $\mathrm{N}$ & $\mathrm{P}$ & $\mathrm{N}$ & $\mathrm{N}$ \\
\hline $\mathrm{T}_{2}$ & $\mathrm{~N}$ & $\mathrm{Z}$ & $\mathrm{P}$ & $\mathrm{N}$ & $\mathrm{P}$ & $\mathrm{P}$ \\
\hline $\mathrm{T}_{3}$ & $\mathrm{~N}$ & $\mathrm{P}$ & $\mathrm{Z}$ & $\mathrm{N}$ & $\mathrm{P}$ & $\mathrm{N}$ \\
\hline $\mathrm{T}_{4}$ & $\mathrm{P}$ & $\mathrm{N}$ & $\mathrm{Z}$ & $\mathrm{P}$ & $\mathrm{N}$ & $\mathrm{P}$ \\
\hline $\mathrm{T}_{5}$ & $\mathrm{Z}$ & $\mathrm{N}$ & $\mathrm{P}$ & $\mathrm{N}$ & $\mathrm{N}$ & $\mathrm{P}$ \\
\hline $\mathrm{T}_{6}$ & $\mathrm{Z}$ & $\mathrm{P}$ & $\mathrm{N}$ & $\mathrm{P}$ & $\mathrm{P}$ & $\mathrm{N}$ \\
\hline$\left(\mathrm{CS}_{\mathrm{a}} \times, \mathrm{CS}_{\mathrm{b}}\right)$ & $\mathrm{P} / \mathrm{N}$ & $\mathrm{P} / \mathrm{N}$ & $\mathrm{P} / \mathrm{N}$ & $\mathrm{P} / \mathrm{N}$ & $\mathrm{Z}$ & $\mathrm{P} / \mathrm{N}$ \\
\hline$\left(\mathrm{CS}_{\mathrm{a}}, \mathrm{CS}_{\mathrm{b}} \times\right)$ & $\mathrm{P} / \mathrm{N}$ & $\mathrm{P} / \mathrm{N}$ & $\mathrm{P} / \mathrm{N}$ & $\mathrm{Z}$ & $\mathrm{P} / \mathrm{N}$ & $\mathrm{P} / \mathrm{N}$ \\
\hline$\left(\mathrm{CS}_{\mathrm{a}} \times, \mathrm{CS}_{\mathrm{c}}\right)$ & $\mathrm{P} / \mathrm{N}$ & $\mathrm{P} / \mathrm{N}$ & $\mathrm{P} / \mathrm{N}$ & $\mathrm{P} / \mathrm{N}$ & $\mathrm{P} / \mathrm{N}$ & $\mathrm{Z}$ \\
\hline$\left(\mathrm{CS}_{\mathrm{a}}, \mathrm{CS}_{\mathrm{c}} \times\right)$ & $\mathrm{P} / \mathrm{N}$ & $\mathrm{P} / \mathrm{N}$ & $\mathrm{P} / \mathrm{N}$ & $\mathrm{Z}$ & $\mathrm{P} / \mathrm{N}$ & $\mathrm{P} / \mathrm{N}$ \\
\hline$\left(\mathrm{CS}_{\mathrm{b}} \times, \mathrm{CS}_{\mathrm{c}}\right)$ & $\mathrm{P} / \mathrm{N}$ & $\mathrm{P} / \mathrm{N}$ & $\mathrm{P} / \mathrm{N}$ & $\mathrm{P} / \mathrm{N}$ & $\mathrm{P} / \mathrm{N}$ & $\mathrm{Z}$ \\
\hline$\left(\mathrm{CS}_{\mathrm{b}}, \mathrm{CS}_{\mathrm{c}} \times\right)$ & $\mathrm{P} / \mathrm{N}$ & $\mathrm{P} / \mathrm{N}$ & $\mathrm{P} / \mathrm{N}$ & $\mathrm{P} / \mathrm{N}$ & $\mathrm{Z}$ & $\mathrm{P} / \mathrm{N}$ \\
\hline
\end{tabular}

TABLE II. EXPERIMENT SPECIFICATION

\begin{tabular}{|c|c|c|}
\hline Parameters & Symbols & Value \\
\hline DC-link voltage & $V_{d c}$ & $400 \mathrm{~V}$ \\
\hline Grid phase voltages & $v_{x N}$ & $110 \mathrm{~V}(\mathrm{rms}), 50 \mathrm{~Hz}$ \\
\hline Rated power & $P_{\text {rate }}$ & $1.2 \mathrm{~kW}$ \\
\hline $\begin{array}{c}\text { Filter inductances in } \\
\text { experiments }\end{array}$ & $L_{f}$ & $9 \mathrm{mH}$ \\
\hline $\begin{array}{c}\text { Equivalent resistances } \\
\text { Switching/Sampling } \\
\text { frequency }\end{array}$ & $R_{f}$ & $0.3 \Omega$ \\
\hline $\begin{array}{c}\text { Sampling error of } V_{d c}, \\
v_{x y}, v_{x N}, i_{x}\end{array}$ & $\sigma_{V d c}, \sigma v_{x y}, \sigma v_{x N}$, & $4 \mathrm{~V}, 4 \mathrm{~V}, 2 \mathrm{~V}$, \\
\hline$\sigma_{i x}$ & $0.06 \mathrm{~A}$ \\
\hline Inductance error & $\sigma_{L f}$ & $1.8 \mathrm{mH}$ \\
\hline Dead time & $T_{D D}$ & $1.5 \mu \mathrm{s}$ \\
\hline Delay time & $T_{D L}$ & $1 \mu \mathrm{s}$ \\
\hline
\end{tabular}

$$
\begin{gathered}
\bar{V}_{X L}{ }^{*}[n]=\frac{1}{2}\left(V_{d c}[n-1]+V_{d c}[n]\right) \cdot d_{x}[n-1] \\
\bar{V}_{N L}{ }^{*}[n]=\frac{1}{6}\left(V_{d c}[n-1]+V_{d c}[n]\right) \cdot \sum_{x=a, b, c} d_{x}[n-1]
\end{gathered}
$$

More detailed derivation of the calculation model can be found in [8].

Finally, the average output voltage deviations are

$$
\begin{aligned}
& \overline{\Delta v}_{x y}[n]=\bar{v}_{x y}{ }^{*}[n]-\bar{v}_{x y}[n] \\
& \overline{\Delta v}_{x N}[n]=\bar{v}_{x N}{ }^{*}[n]-\bar{v}_{x N}[n]
\end{aligned}
$$

\section{B. Calculate Thresholds}

Due to calculation error from measure error, parameter error, dead time, delay and so on, thresholds $\overline{\Delta v}_{t h, x y}[n]$ and $\overline{\Delta v}_{t h, x N}[n]$ are needed to avoid false alarm caused by these calculation error. The error-adaptive method proposed in [8] is applied to determine the thresholds. 


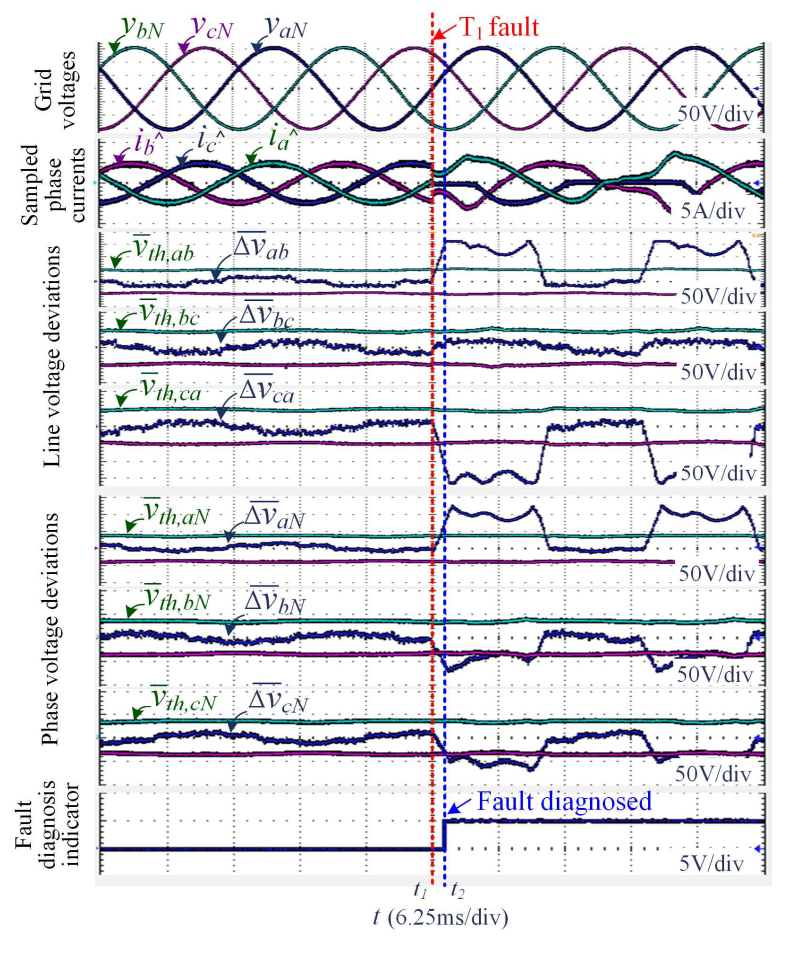

Fig. 5. Experimental result of $T_{1}$ fault diagnosis

Maximum calculation error of diagnosis variables from sampling error $\sigma_{V d c}, \sigma_{v x y}, \sigma_{v x N}, \sigma_{i x}$ and parameter error $\sigma_{L f}$ are

$$
\begin{aligned}
& \xi_{\overline{\Delta v_{x y}}, S P}[n]=\frac{1}{T_{S}} \sigma_{L_{f}}\left(\mid i_{x}[n]-i_{x}[n-1]\right)+\frac{1}{T_{S}} \sigma_{L_{f}}\left(\mid i_{y}[n]-i_{y}[n-1]\right) \\
& +\sigma_{V_{d c}}\left(\mid d_{x}[n-1]-d_{y}[n-1]\right)+\sigma_{v_{x y}}+\frac{4}{T_{S}} \sigma_{i_{x}} L_{f} \\
& \xi_{\overline{\Delta v_{x N}}, S P}[n]=\frac{1}{T_{S}} \sigma_{L_{f}}\left(\mid i_{x}[n]-i_{x}[n-1]\right)+\sigma_{V_{d c}}\left(\mid d_{x}[n-1]\right. \\
& \left.\quad-\frac{1}{3}\left(d_{a}[n-1]+d_{b}[n-1]+d_{c}[n-1]\right) \mid\right)+\sigma_{v_{x N}}+\frac{2}{T_{S}} \sigma_{i_{x}} L_{f}
\end{aligned}
$$

The determination of sampling errors and inductance error is explained in [19].

Maximum calculation errors from dead time $T_{D D}$ and delay time $T_{D L}$ are

$$
\begin{gathered}
\xi_{\overline{\Delta v}_{x y}, D D}=2 V_{d c} \cdot \frac{T_{D D}}{T_{S}}, \xi_{\overline{\Delta v_{x N}, D D}}=\frac{4}{3} V_{d c} \cdot \frac{T_{D D}}{T_{S}} \\
\xi_{\overline{\Delta v}_{x y}, D L}=2 V_{d c} \cdot \frac{T_{D L}}{T_{S}}, \xi_{{\overline{\Delta v_{x N}}, D L}}=2 V_{d c} \cdot \frac{T_{D L}}{T_{S}}
\end{gathered}
$$

Finally, the thresholds can be selected as

$$
\begin{aligned}
& \overline{\Delta v}_{t h, x y}=\xi_{\overline{\Delta v}_{x y}, S P}+\xi_{\overline{\Delta v}_{x y}, D D}+\xi_{\overline{\Delta v}_{x y}, D L} \\
& \overline{\Delta v}_{t h, x N}=\xi_{\overline{\Delta v}_{x N}, S P}+\xi_{\overline{\Delta v}_{x N}, D D}+\xi_{\overline{\Delta v}_{x N}, D L}
\end{aligned}
$$

\section{Locate Fault}

With average output voltage deviations and thresholds, the voltage deviation polarities can be generated as

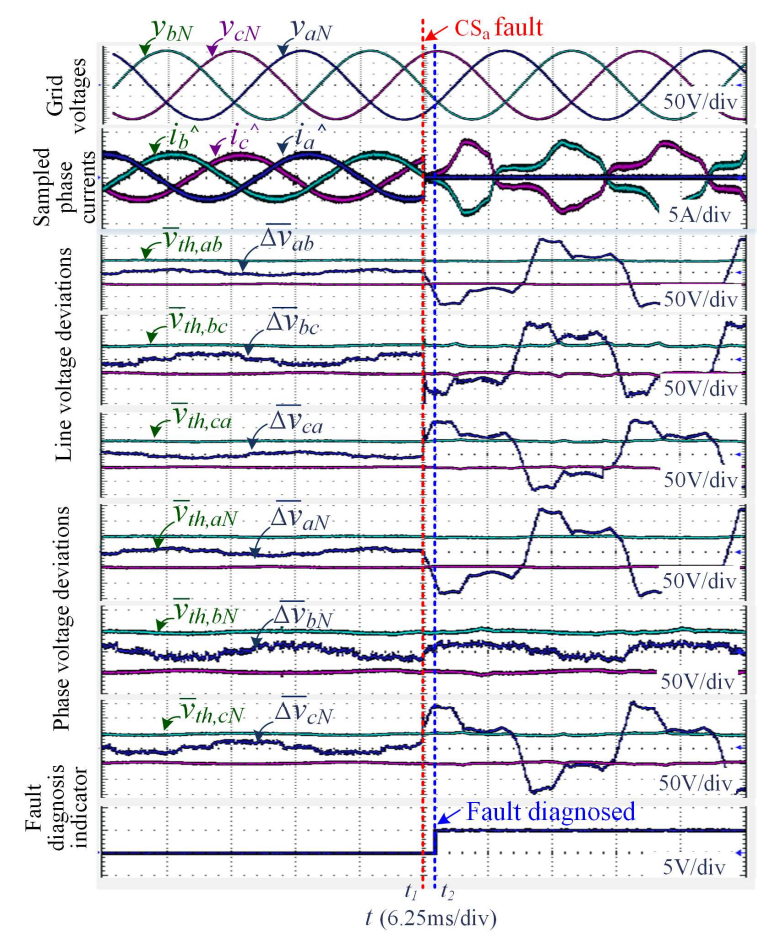

Fig. 6. Experimental result of $\mathrm{CS}_{\mathrm{a}}$ fault diagnosis

$$
\Delta_{\text {sub }}=\left\{\begin{array}{l}
P, \quad \Delta V_{\text {sub }} \geq \Delta V_{t h, s u b} \\
Z,-\Delta V_{t h, s u b}<\Delta V_{\text {sub }}<\Delta V_{t h, s u b} \\
N, \quad \Delta V_{\text {sub }} \leq-\Delta V_{t h, s u b}
\end{array}\right.
$$

Then, according to the faulty characteristics shown in TABLE I, the criteria for diagnosing IGBT OC fault and current sensor fault are given in TABLE II. In order to further improve robustness against disturbances, the minimum time judging rule is implemented in the method. The fault diagnosis result have to remain for the minimum time $T_{\min }$ to be considered reliable. Higher $T_{\min }$ leads to better robustness and meanwhile longer detection time. In this paper, $T_{\min }$ is set as $2 T_{S}$.

\section{EXPERIMENTAL RESULTS}

In order to verify the effectiveness of the method, experiments have been carried out on OPAL-RT 4510 platform. The experiment specification is shown in TABLE III. The experimental results of $\mathrm{T}_{1} \mathrm{OC}$ fault and $\mathrm{CS}_{\mathrm{a}}$ fault are shown in Fig. 5 and Fig. 6 respectively.

In Fig.5, before the fault comes, all the diagnosis variables are within thresholds. After the fault is triggered at $t_{l}$, the voltage deviation polarities $\left(\Delta_{a b}, \Delta_{b c}, \Delta_{c a}, \Delta_{a N}, \Delta_{b N}, \Delta_{c N}\right)$ change to $(\mathrm{P}, \mathrm{Z}, \mathrm{N}, \mathrm{P}, \mathrm{N}, \mathrm{N})$ soon. According to criteria in TABLE II, the $\mathrm{T}_{1}$ fault is diagnosed at $t_{2}$.

In Fig.6, after the current sensor fault is triggered at $t_{l}$, the voltage deviation polarities change to $(\mathrm{N}, \mathrm{N}, \mathrm{P}, \mathrm{N}, \mathrm{Z}, \mathrm{P})$ soon. According to criteria in TABLE II, the $\mathrm{CS}_{\mathrm{a}}$ fault is diagnosed at $t_{2}$.

These experiments verify that the proposed method can diagnose the IGBT faults and current sensor faults effectively. 


\section{CONCLusions}

A novel diagnosis method based on average output voltage deviations is proposed in the paper. This method can diagnose both IGBT OC faults and current sensor faults quickly in gridtied 3P3W inverters with two current sensors by utilizing signals available in the controller. The fault analysis, method principle and model calculations are introduced. Finally, the effectiveness of the proposed method is verified by experiments.

\section{REFERENCES}

[1] S. Yang, A. Bryant, et al., "An industry-based survey of reliability in power electronic converters," IEEE Trans. Ind. Appl., vol. 47, no. 3, pp. 1441-1451, May-June 2011

[2] Y. Wang, Z. Li, M. Xu, et al., "A comparative study of two diagnostic methods based on switching voltage pattern for IGBTs open-circuit faults in voltage-source inverters," J. Power Electron., vol. 16, no. 3, pp. 1087-1096, 2016

[3] Z. Li, Y. Wang, H. Ma, L. Hong, "Open-transistor faults diagnosis in voltage-source inverter based on phase voltages with sliding-window counting method". in Proc. 42nd IEEE IECON., pp. 435-440, 2016.

[4] Wang Y, Li Z, Lin L. "A novel diagnosis method based on flexible error voltage for IGBTs open-circuit faults in voltage-source inverters", in Proc. 41st IEEE IECON, pp. 19-24, 2015

[5] Estima, J.O. and A.J. Marques Cardoso, "A New Approach for RealTime Multiple Open-Circuit Fault Diagnosis in Voltage-Source Inverters", IEEE Trans. Ind. Appl., vol. 47, no. 6, pp. 2487-2494, Nov. 2011

[6] Estima J O, Cardoso A J M., “A new algorithm for real-time multiple open-circuit fault diagnosis in voltage-fed PWM motor drives by the reference current errors", IEEE Trans. Ind. Electron., vol. 60, no. 8, pp. 3496-3505, Aug. 2013.

[7] Choi J H, Kim S, Yoo D S, et al., "A diagnostic method of simultaneous open-switch faults in inverter-fed linear induction motor drive for reliability enhancement", IEEE Trans. Ind. Electron., vol. 62, no. 7, pp. 4065-4077, July 2015
[8] Z. Li, H. Ma, Z. Bai, Y. Wang and B. Wang, "Fast transistor opencircuit faults diagnosis in grid-tied three-phase VSIs based on average bridge arm pole-to-pole voltages and error-adaptive thresholds", IEEE Trans. Power Electron., vol. 33, no. 9, pp. 8040-8051, Sept. 2018.

[9] An Q T, Sun L, Sun L Z., "Current residual vector-based open-switch fault diagnosis of inverters in PMSM drive systems", IEEE Trans. Power Electron., vol. 30, no. 5 pp. 2814-2827, May 2015.

[10] Jlassi I, Estima J O, El Khil S K, et al., "Multiple open-circuit faults diagnosis in back-to-back converters of PMSG drives for wind turbine systems", IEEE Trans. Power Electron., vol. 30, no. 5, pp. 2689-2702, May 2015.

[11] Yetendje, Alain, et al., "Sensor fault tolerant control of a magnetic levitation system." International Journal of Robust and Nonlinear Control, vol. 20, no.18, pp. 2108-2121, Nov. 2010

[12] Youssef A B, El Khil S K, Slama-Belkhodja I., "State observer-based sensor fault detection and isolation, and fault tolerant control of a single-phase PWM rectifier for electric railway traction", IEEE Trans. Power Electron., vol. 28, no. 12, pp. 5842-5853, May. 2013.

[13] Papadopoulos P M, Hadjidemetriou L, Kyriakides E, et al., "Robust fault detection, isolation, and accommodation of current sensors in grid side converters", IEEE Trans. Ind. Appl., vol. 53, no. 3, pp. 2852-2861, Dec. 2017

[14] Yu Y, Zhao Y, Wang B, et al., "Current sensor fault diagnosis and tolerant control for VSI-based induction motor drives", IEEE Trans. Power Electron., vol. 33, no. 5, pp. 4238-4248, May 2018.

[15] El Khil S K, Jlassi I, Estima J O, et al. "Current sensor fault detection and isolation method for PMSM drives using average normalised currents", Electronics Letters, vol. 52, no. 17, pp. 1434-1436, July 2016.

[16] Gou B, Ge X L, Liu Y C, et al., "Load-current-based current sensor fault diagnosis and tolerant control scheme for traction inverters", Electronics Letters, vol. 52, no. 20, pp. 1717-1719, Sept. 2016.

[17] Jlassi, Imed, et al., "A robust observer-based method for IGBTs and current sensors fault diagnosis in voltage-source inverters of PMSM drives," IEEE Trans. Ind. Appl., vol. 53, no. 3, pp. 2894-2905. MayJune 2017

[18] Y. Ren, Z. Li, B. Wang, Z. Bai and H. Ma, “A fast average modelbased method for IGBT and current sensor fault diagnosis in grid-tied inverters," in Proc. 44th IEEE IECON, pp. 3809-3814, 2018.

[19] Z. Li, B. Wang, Y. Ren, et al.,"L and LCL filtered grid-tied singlephase inverter transistor open-circuit fault diagnosis based on post-fault reconfiguration algorithms", IEEE Trans. Power Electron., 2018, in press: DOI 10.1109/TPEL.2019.2891249 